\title{
Correction to: Preparation and characterization of bio-based green renewable composites from poly(lactic acid) reinforced with corn stover
}

\author{
Jipeng Guo ${ }^{1}$ Chi-Hui Tsou ${ }^{1,2,3,4,5,6}$ - Manuel Reyes De Guzman ${ }^{1,4,5}$. Chin-San Wu' ${ }^{7}$ Xuemei Zhang ${ }^{1}$. \\ Zhujun Chen $^{1} \cdot$ Yi-Hua Wen ${ }^{1} \cdot$ Tao Yang $^{1} \cdot$ Yong-Jie Zhuang ${ }^{1} \cdot$ Feifan $\mathrm{Ge}^{1} \cdot$ Zhijun Chen $^{1} \cdot$ Zhaohua Wang $^{1}$
}

Published online: 13 October 2021

(C) The Polymer Society, Taipei 2021

Correction to: Journal of Polymer Research (2021) 28:199 https://doi.org/10.1007/s10965-021-02559-1

The original version of this article unfortunately contained a mistake. The first author "Jipeng Guo" was not affiliated to any affiliation in the originally published article. "Jipeng Guo" must be affiliated to affiliation 1 as shown here in this correction paper.

The original article has been corrected as well.

The original article can be found online at https://doi.org/10.1007/ s10965-021-02559-1.

Chi-Hui Tsou

mayko0301@hotmail.com

$\triangle$ Xuemei Zhang

798538268@zju.edu.cn

1 Material Corrosion and Protection Key Laboratory of Sichuan Province, School of Materials Science and Engineering, Sichuan University of Science and Engineering, Zigong 643000, China

2 Sichuan Yibin Plastic Packaging Materials Co. Ltd, Yibin 644007, China

3 Sichuan Golden-Elephant Sincerity Chemical Co. Ltd, Meishan 620010, China

4 Sichuan Zhixiangyi Technology Co. Ltd, Chengdu 610051, China

5 Sichuan Zhirenfa Biotechnology Co. Ltd, Zigong 643000, China

6 Center of Excellence in Textiles, Department of Materials Science, Faculty of Science, Chulalongkorn University, Bangkok 10330, Thailand

7 Department of Applied Cosmetology, Kao Yuan University, Kaohsiung County, Fongshan 82101, Taiwan
Publisher's Note Springer Nature remains neutral with regard to jurisdictional claims in published maps and institutional affiliations. 